\title{
Von Netzalter, Wasserverlusten und Schadensraten zur langfristigen Erneuerungsplanung
}

\author{
Roman Neunteufel · Ernest Mayr · Stefan Krakow · Laurent Richard · Ralf Herda
}

Online publiziert: 29. März 2017

(C) Der/die Autor(en) 2017. Dieser Artikel ist eine Open-Access-Publikation.

\begin{abstract}
Zusammenfassung Die Planung bzw. Festlegung einer langfristigen Erneuerungsstrategie für Trinkwasserversorgungsanlagen ist ein oft diskutiertes Thema und grundsätzlich keine einfache Aufgabe. So gibt es zum Beispiel auf der einen Seite die technische Nutzungsdauer von Trinkwasserrohrleitungen, die unter anderem von der Materialwahl, dem Durchmesser und den Untergrundverhältnissen abhängt, und auf der anderen Seite existieren betriebliche Zwänge, wie die gemeinsame Koordinierung von Baumaßnahmen mit anderen Leitungsträgern und/oder dem Straßenbau, die zu einer vorzeitigen Auswechslung von Rohrleitungen führen können.

Für die Festlegung einer nachhaltigen und zufriedenstellenden Rehabilitationsplanung sollte der Zustand der Anlagen in Verbindung mit dem Rohrnetzalter genauer betrachtet werden. Für die Zustandsbewertung lassen sich beispielsweise die Wasserverluste und die vorhandenen Schadensraten heranziehen.
\end{abstract}

DI R. Neunteufel $(\bowtie) \cdot$ DI E. Mayr Institut für Siedlungswasserbau, Industriewasserwirtschaft und Gewässerschutz, Universität für Bodenkultur Wien, Muthgasse 18, 1190 Wien, Österreich roman.neunteufel@boku.ac.at

\section{S. Krakow}

Wasserplan - Fischer \&

Herda Ziviltechniker GmbH, Harrachgasse 4, 6845 Hohenems, Österreich

\section{L. Richard}

Kooperative Wasser (Ingenieurbüro | Entwicklung | Plattform), Lenaugasse 2/7, 1080 Wien, Österreich

\section{R. Herda}

Institut für Siedlungswasserwirtschaft und Landschaftswasserbau,

Technische Universität Graz,

Stremayrgasse 10/I, 8010 Graz, Österreich
Des Weiteren spielt die Siedlungsstruktur (z. B. ländlicher Bereich bzw. städtischer Bereich) hinsichtlich der Anlagenbelastungen eine wesentliche Rolle (z. B. Verkehrslasten, Grabungsaktivitäten) für die tatsächlichen Nutzungsdauern. Außerdem hat die Siedlungsstruktur entsprechende Auswirkungen auf die Rehabilitationskosten der Rohrleitungen.

Im Beitrag wird dargestellt, wie bei der Rehabilitationsplanung die Zustandsbewertung der Anlagen einfließen kann. Welche Einflussfaktoren dabei zu beachten sind, wird anhand von aktuellen Erkenntnissen aus dem ÖVGW-Benchmarking hinsichtlich Netzalter, Wasserverluste und Schadensraten gezeigt. Anhand eines Beispiels der endgültigen Prioritätenreihung für die Erneuerungsplanung wird klar, dass auch die jeweilige Wichtigkeit eines Systemteils hinsichtlich der Versorgungssicherheit für die Planung eine Rolle spielt.

\section{From grid age, water loss and pipe damage rates to long-term rehabilitation planning}

Abstract Though planning/defining a long-term rehabilitation strategy for drinking-water supply facilities is a frequently discussed topic, it also represents a daunting challenge. Various factors have to be taken into account, e.g. the longevity of drinking-water distribution network - which is determined by e.g. the choice of material, pipe diameter and underground conditions - on the one hand; and by operational constraints - e.g. the need to coordinate planned measures with other service providers and/or the road works, which can result in pipe sections being replaced sooner than planned on the other.

In order to achieve sensible and sustainable rehabilitation planning, the current status of the facilities and distribution network must be carefully assessed. In this regard, aspects such as water loss and failure rates could be used as a basis for status appraisals.

Furthermore, the municipal structure (e.g. urban or rural) significantly impacts the strain on facilities (e.g. through excavations, increased traffic, etc.), and accordingly, on their actual service lifespans. The specific distribution network structure also significantly impacts the cost of rehabilitation.

The paper shows how the assessment of infrastructure current status can be used for better rehabilitation planning. On the basis of the latest insights gleaned from the ÖVGW benchmarking regarding network age, water loss and failure rates, it shows which influencing factors should be kept in mind. Finally, the example of a prioritization plan for rehabilitation strategy clearly demonstrates that the importance of a specific system component is also relevant for session making.

\section{Hintergrund und Zielsetzung}

Seit langem wird im Kreis der Wasserversorger über den nötigen Umfang der Rehabilitationsmaßnahmen und deren Finanzierung in Zusammenhang mit einem kostendeckenden Wasserpreis diskutiert.

Natürlich sollte nicht alleine aufgrund des Alters von Leitungsnetzen oder Netzteilen eine bestimmte Rehabilitationsrate festgelegt werden. Wichtig ist, den Zustand des Leitungsnetzes zu kennen und in die Erneuerungsplanung miteinfließen zu lassen. Für die Wasserversorgungsnetze stehen dazu im Wesentlichen zwei Kennzahlen-Kategorien zur Verfügung: die Wasserverluste und die Schadenszahlen. Ein weiterer Zusammenhang besteht zwischen den Schadensraten und der Intensität der durchgeführten Lecksuche und somit auch zu den Wasserverlusten.

Nichtsdestotrotz steht das Netzalter mit den Verlusten und den Schadenszahlen in einem eindeutig nachweisbaren Zusammenhang. Aufgrund der Langlebigkeit der Wasserversorgungs- 
Tab. 1 Strukturkategorien von Wasserversorgungsnetzen

\begin{tabular}{|c|c|c|c|}
\hline & $\begin{array}{l}\text { Strukturkategorie } 1 \\
\text { „ländlich“ }\end{array}$ & $\begin{array}{l}\text { Strukturkategorie } 2 \\
\text { „städtisch“ }\end{array}$ & $\begin{array}{l}\text { Strukturkategorie } 3 \\
\text { "großstädtisch“" }\end{array}$ \\
\hline $\begin{array}{l}\text { Einflussfaktoren Nut- } \\
\text { zungsdauer: } \\
\text { Dynamische und statische Las- } \\
\text { ten, Beschädigungen durch } \\
\text { Dritte }\end{array}$ & $\begin{array}{l}\text { „geringe Schadensraten, hö- } \\
\text { here Nutzungsdauer" } \\
\text { geringe Geländeauflasten; keine/kaum } \\
\text { Bebauung im Einflussbereich, geringe } \\
\text { Setzungen; keine/kaum Verkehrslasten } \\
\text { im Einflussbereich }\end{array}$ & $\begin{array}{l}\text { „durchschnittliche Schadens- } \\
\text { raten und Nutzungsdauer“ } \\
\text { teilweise bis flächige Bebauung; } \\
\text { häufige Verkehrslasten im Einzugs- } \\
\text { bereich; wenig sonstige Leitungsträger } \\
\text { im Nahbereich }\end{array}$ & $\begin{array}{l}\text { „höhere Schadensraten und } \\
\text { ev. eingeschränkte Nutzungs- } \\
\text { dauer“ } \\
\text { flächige Bebauung auch mit großen } \\
\text { Gebäuden; ständige Verkehrslasten } \\
\text { im Einzugsbereich; viele sonstige } \\
\text { Leitungsträger im Nahbereich }\end{array}$ \\
\hline $\begin{array}{l}\text { Einflussfaktoren Rehabili- } \\
\text { tationskosten: } \\
\text { Bodenversiegelung, Zugänglich- } \\
\text { keit der Baustelle, Verkehrs- } \\
\text { behinderung }\end{array}$ & $\begin{array}{l}\text { "geringe Kosten“ } \\
\text { nur teilweise oder normale } \\
\text { Oberflächenversiegelung; leichte Zu- } \\
\text { gänglichkeit, keine Behinderungen, } \\
\text { Baustofflagerung/Aushublagerung } \\
\text { möglich }\end{array}$ & $\begin{array}{l}\text { "durchschnittliche Kosten“ } \\
\text { Flächendeckende, normale } \\
\text { Oberflächenversiegelung; Zugänglich- } \\
\text { keit leicht eingeschränkt, Baustofflage- } \\
\text { rung, Aushublagerung problematisch }\end{array}$ & $\begin{array}{l}\text { „hohe Kosten“ } \\
\text { Ev. spezielle Oberflächenversiege- } \\
\text { lung (Pflaster) Baustofflagerung, } \\
\text { Aushublagerung nicht möglich; Ver- } \\
\text { kehrsbehinderungen, Zugänglichkeit } \\
\text { eingeschränkt }\end{array}$ \\
\hline
\end{tabular}

netze und der Tatsache, dass ein Großteil der österreichischen Leitungsnetze in einem sehr guten Zustand ist, wird die Notwendigkeit der langfristigen Erneuerungsplanung leicht übersehen.

Ziel ist es, die Zusammenhänge vom Zeitpunkt der Hauptbauphasen der Infrastruktur, deren derzeitigem Zustand und den anstehenden Erneuerungen bewusst zu machen. Nicht-Ziel ist es, einen Ersatz für eine detaillierte Rehabilitationsplanung und Prioritätenreihung zu schaffen. Dazu sind die Überlegungen zur langfristigen (strategischen) Erneuerungsplanung nicht in der Lage.

\section{Theoretische Überlegungen}

\subsection{Nutzungsdauer und} Reinvestitionserfordernis

Technisch gesehen können Leitungsmaterial, Rohrdimension, das jeweilige Baujahr (Rohrcharge), Dichtungen, die Bodenart, die Verlegeart, die Lagerung vor dem Einbau, Betriebsparameter (Druck und Druckstöße) und die Struktur des Versorgungsgebiets einen Einfluss auf die Nutzungsdauer haben.

Unter der Struktur des Versorgungsgebiets (Strukturkategorien entsprechend dem ÖVGW-Benchmarking: siehe Tab. 1) ist die Urbanität zu verstehen. Dabei kann in ländliche, städtische und großstädtische Versorgungsstrukturen unterschieden werden. Mittels der Differenzierung in Strukturkategorien kann die jeweils auftretende Belastung des Rohrmaterials durch Bodenspannungen (z. B. Gebäudelasten, Verkehrslasten, Aufgrabungen) nachvollzogen werden. Die Strukturkategorie oder Urbanität eines Netzteiles kann in weiterer Folge auch zur Kostenschätzung der zu erneuernden Leitungslängen herangezogen werden. Somit kann eine Erneuerungsplanung auch gleich mit einem Kostenplan ergänzt werden.

Ein weiterer Faktor, der bei Überlegungen zur Nutzungsdauer von Wasserleitungsnetzen relevant ist, ist die vorzeitige Auswechslung aufgrund anderer baulicher Maßnahmen. Durch die Möglichkeit oder Notwendigkeit der koordinierten Mitverlegung werden Leitungsteile auch getauscht, wenn diese noch nicht besonders nahe an ihrer erwartbaren Lebensdauer sind. Speziell, wenn dadurch insgesamt Kosten gespart werden können. Letztendlich sind vorzeitige Auswechslungen also wirtschaftliche Entscheidungen.

Die tatsächliche Nutzungsdauer kann somit von sehr vielen Faktoren abhängen. Dadurch ist die Nutzungsdauer eines neugebauten Rohrstrangs oder eines Netzteils nicht von vornherein bekannt, sondern wird erst im Laufe der Zeit erkennbar. Die Beobachtung der Entwicklung der Wasserverluste und der auftretenden Schäden ist daher von großer Bedeutung. Letztendlich und langfristig betrachtet ergibt sich eine durchschnittlich nötige, jährliche Erneuerungsrate, die direkt aus der durchschnittlichen Nutzungsdauer der Leitungsnetze abgeleitet werden kann.

In Abb. 1 ist beispielhaft eine Langzeitplanung für die Erneuerung einer einmaligen Investition dargestellt. Auf einen Zeitmaßstab wird dabei bewusst verzichtet, da die durchschnittliche Nutzungsdauer der Investition anhand individueller Erfahrungswerte angenommen werden muss und entsprechend den Einflussfaktoren für verschiedene Netzteile (bzw. Leitungsgruppen oder Bauteile) unterschiedlich ist. Das Rehabilitationserfordernis ergibt sich dabei rund um den Zeitpunkt der durchschnittlichen Lebensdauer. Teile des Leitungsnetzes müssen schon früher erneuert werden, andere Teile können länger in Verwendung bleiben und müssen erst später erneuert werden. Daraus ergibt sich eine typische „Erneuerungswelle“. Ein weiteres Maximum des Reinvestitionserfordernisses zeigt sich, wenn die Infrastruktur bereits zum zweiten Mal erneuert werden muss. Das Maximum ist dabei bereits weniger stark ausgeprägt, da über kürzere und längere Lebensdauern von Teilen des Leitungsnetzes eine Vergleichmäßigung stattfindet. Dafür sind auch keine so deutlichen Minima mehr vorzufinden. Langfristig stellt sich so ein durchschnittlich nötiges Reinvestitionserfordernis ein.

Diese Betrachtungsweise ist zwar sehr anschaulich, gilt aber nur für eine Einzelinvestition. Der Bauzeitpunkt eines Wasserversorgungssystems ist hingegen selten auf eine einzige Bauphase beschränkt und zudem setzen sich die Netze im Allgemeinen aus einem Materialmix zusammen, wodurch unterschiedliche Nutzungsdauern entstehen. Um eine von der jeweils individuellen Nutzungsdauer entkoppelte Maßzahl zur Beurteilung des Netzalters zur Verfügung zu haben, kann die Netzalterquote verwendet werden.

\subsection{Die Netzalterquote (NAX)}

Die Altersquote einer Leitung ist als verstrichene Zeit der erwartbaren Nutzungsdauer definiert. Direkt nach der Errichtung hat ein Leitungsteil eine Altersquote von $0 \%$. Nach der halben erwartbaren Nutzungsdauer beträgt die Altersquote $50 \%$. Bei Erreichen von $100 \%$ muss (im Durchschnitt) die Erneuerung erfolgen.

Die Netzalterquote einer Materialgruppe (oder Leitungsgruppe) errechnet sich durch die längengewichtete Durchschnittsbildung aller einzelnen 


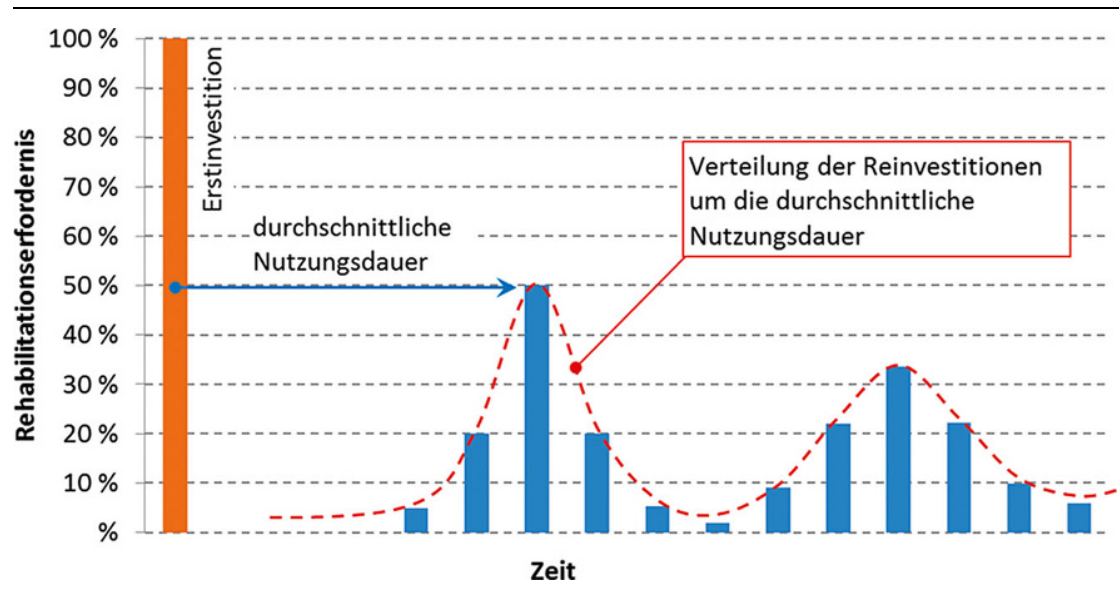

Abb. 1 Beispielhafte Langzeitplanung der Rehabilitation

Leitungen der Gruppe. Die durchschnittliche Netzalterquote des gesamten Leitungsnetzes kann dann durch eine längengewichtete Durchschnittsbildung der einzelnen Materialgruppen (oder Leitungsgruppen) errechnet werden (Neunteufel et al. 2008).

Um eine vergleichbare Indexzahl $\mathrm{zu}$ errechnen, die ein durchschnittliches Alter des Leitungsnetzes zeigt, ist es nötig, ein vereinheitlichtes Referenzalter für die Nutzungsdauer verschiedener Materialien zu schaffen. Dazu wurde im ÖVGW-Arbeitskreis Benchmarking mit Vertretern verschiedener Wasserwerke für jedes Material ein vereinheitlichtes Referenzalter festgelegt, selbst wenn dieses für die einzelnen Betriebe deutlich abweichen kann.

Für die Berechnung der Netzalterquote ist neben der jeweiligen Länge zu jeder Materialgruppe (oder Leitungsgruppe) das aktuelle durchschnittliche Alter und das Referenzalter (erwartbare Lebensdauer) nötig.

Die Theorie besagt, dass sich bei unterschiedlicher Altersstruktur des Netzes bzw. nach langfristigem Betrieb des Leitungsnetzes, samt rechtzeitiger Erneuerung, eine Netzalterquote von rund $50 \%$ einstellen müsste. Diese $50 \%$ ergeben sich zum Beispiel aus einer ausgewogenen Mischung ganz neuer Leitungen ( $0 \%$ der zu erwarteten Nutzungsdauer verbraucht), neueren Leitungen, älteren Leitungen und ganz alten Leitungen (100\% der zu erwarteten Nutzungsdauer verbraucht), die als nächstes zu erneuern sind und danach wieder bei $0 \%$ anfangen.

Um die Leitungsnetze für Vergleiche in Gruppen zusammenfassen zu können, wurden im ÖVGW-Benchmarking Altersgrenzen von NAX $<40 \%$ für junge Netze und NAX $>60 \%$ für alte Netze definiert.

Ein hoher Wert (NAX deutlich > $60 \%$ ) deutet auf eine Überalterung des Leitungsnetzes und verstärktes Rehabilitationserfordernis hin. Zwar könnten Rohrleitungen unter günstigen Bedingungen auch weit über das angenommene Referenzjahr funktionsfähig bleiben, es ist aber nicht der Regelfall.

Sollten die Leitungen eine eher einheitliche Altersstruktur aufweisen, dann ist damit $\mathrm{zu}$ rechnen, dass ein großer Teil des Netzes gleichzeitig den Zeitpunkt zur Erneuerung erreicht und innerhalb kurzer Zeit große Reinvestitionen anstehen (vgl. Abb. 1).

\section{3 iNAX}

iNAX steht für eine individuelle Netzalterquote. Jeder Wasserversorger kann aus seinen Erfahrungen für jedes Leitungsmaterial, jeden Durchmesser, jeden Bauzeitpunkt bzw. jede Rohrcharge, jede Bodenart und jede Strukturkategorie gesonderte Überlegungen zur Nutzungsdauer der Infrastruktur anstellen. Daraus ergeben sich individuelle Referenzalter. Die Leitungen werden dazu am besten zu Leitungsgruppen zusammengefasst. Danach kann für jede Leitungsgruppe die Netzalterquote berechnet werden.

Das ist natürlich mit einem hohen Aufwand verbunden und oft stehen bei neu gebauten Netzteilen oder nach Materialumstellungen noch keine Erfahrungen zur Verfügung. Dann muss auf Erfahrungen anderer WVU bzw. auf Durchschnittswerte zurückgegriffen werden. Die Berechnung einer individuellen Netzalterquote ist anhand der Leitungsgruppen auch für Teile des Leitungsnetzes möglich und hat jedenfalls den Vorteil der Anpassung auf die lokalen Verhältnisse und eigenen Einschätzung der wahrscheinlichen Nutzungsdauer.

\section{Empirische Ergebnisse}

\subsection{Netzstrukturparameter}

Wie bereits in Tab. 1 gezeigt wurde, können Wasserleitungsnetze grob in drei Strukturkategorien eingeteilt werden. Die im ÖVGW-Benchmarking verwendete, typische Charakteristik der Strukturgruppen ist in Tab. 2 dargestellt. Für den Fall, dass die spezifische Netzabgabe, die Hausanschlussdichte und die spezifische Zählerabgabe auf verschiedene Strukturgruppen hindeuten, kann nach dem Mehrheitsprinzip vorgegangen werden. Im Zweifelsfall ist die spezifische Netzabgabe maßgeblich. Im DVGW-Arbeitsblatt W392 (2003) werden die Versorgungsstrukturen zum Beispiel nur nach der spezifischen Netzabgabe kategorisiert.

Reine Fernversorger oder WVU mit großen Abgabemengen an Weitervertei-

Tab. 2 Netzstrukturparameter entsprechend dem ÖVGW-Benchmarking

\begin{tabular}{|c|c|c|c|}
\hline & $\begin{array}{l}\text { Strukturkategorie } 1 \\
\text { „ländlich“ }\end{array}$ & $\begin{array}{l}\text { Strukturkategorie } 2 \\
\text { „städtisch“ }\end{array}$ & $\begin{array}{l}\text { Strukturkategorie } 3 \\
\text { "großstädtisch" }\end{array}$ \\
\hline Spezifische Netzabgabe * & $\begin{array}{l}<5.000 \mathrm{~m}^{3} \\
\text { pro } \mathrm{km} \text { und Jahr }\end{array}$ & $\begin{array}{l}5.000 \text { bis } 15.000 \mathrm{~m}^{3} \\
\text { pro km und Jahr }\end{array}$ & $\begin{array}{l}>15.000 \mathrm{~m}^{3} \\
\text { pro km und Jahr }\end{array}$ \\
\hline Hausanschlussdichte * & $<20$ Anschlussleitungen pro km & 20 bis 40 Anschlussleitungen pro km & $>40$ Anschlussleitungen pro km \\
\hline Spezifische Zählerabgabe ** & $<250 \mathrm{~m}^{3}$ pro Kundenzähler und Jahr & $\begin{array}{l}250 \text { bis } 500 \mathrm{~m}^{3} \text { pro Kundenzähler und } \\
\text { Jahr }\end{array}$ & $>500 \mathrm{~m}^{3}$ pro Kundenzähler und Jahr \\
\hline
\end{tabular}




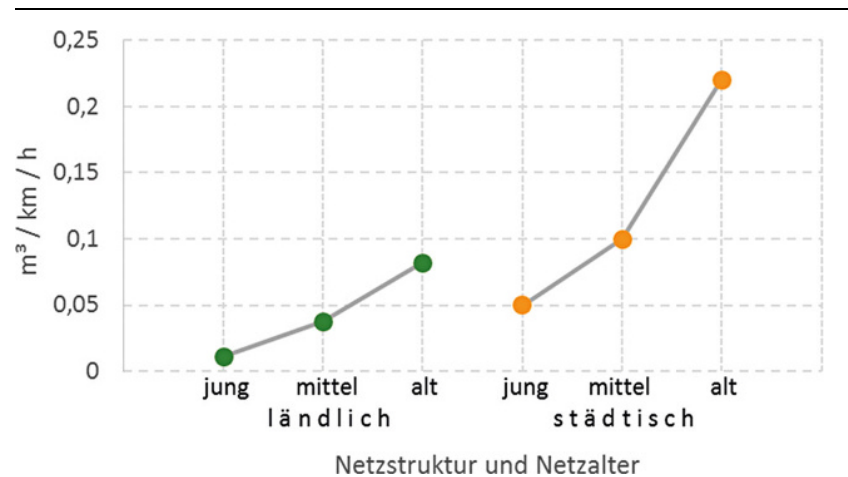

Abb. 2 MittlererealeWasserverluste(Daten:ÖVGW-Benchmarking)

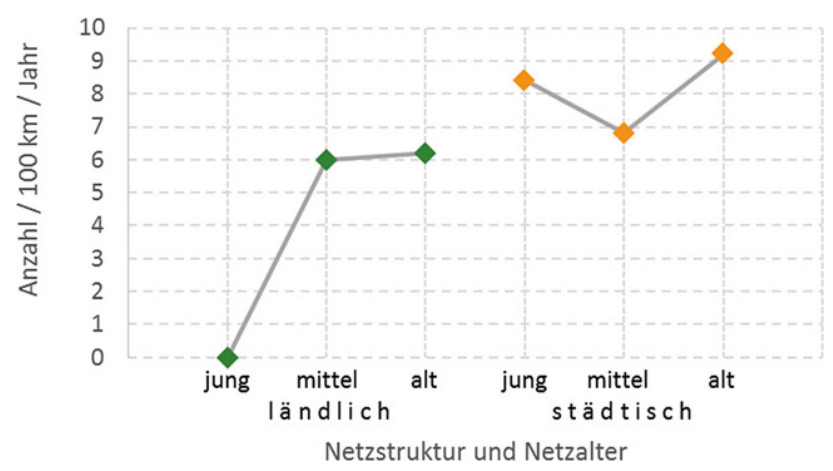

Abb. 3 Mittlere Schadensraten (Daten: ÖVGW-Benchmarking)

ler fallen nicht in die in Tab. 2 genannten Kategorien und sind als gesonderte Strukturkategorie anzusehen. Wenn für WVU mit großen Abgabemengen an Weiterverteiler die Abgabemengen an Direktversorgte herausgerechnet werden können, ist eine Kategorisierung nach Tab. 2 indes möglich.

\subsection{Wasserverluste}

Wie bereits einleitend erwähnt, sollte eine bestimmte Rehabilitationsrate nicht alleine aufgrund des Alters von Leitungsnetzen oder Netzteilen festgelegt werden. Für die Beurteilung des Zustands eines Leitungsnetzes zur Erneuerungsplanung sind neben den Schadenszahlen ebenso die Wasserverluste maßgeblich. Dazu ist es aber wichtig, die Bewertung der aktuellen eigenen Wasserverluste nur gegenüber Vergleichspartnern vorzunehmen, die ähnliche Rahmenbedingungen aufweisen.

Die Wasserverluste unterliegen im Wesentlichen zwei Einflussfaktoren: dem Netzalter (ausgedrückt über die Netzalterquote NAX) und der Netzstruktur (Netzstrukturparameter siehe Tab. 2).
Werden die empirisch verfügbaren Daten aus den ÖVGW-BenchmarkingProjekten nach diesen beiden Einflussfaktoren gruppiert (Clusterung), wird der Zusammenhang klar ersichtlich. Abb. 2 zeigt exemplarisch die Mediane der realen Wasserverluste von ländlich bzw. städtisch strukturierten Wasserversorgungsnetzen entsprechend ihrem Alter. Weitere Strukturgruppen wären großstädtische Leitungsnetze und reine Fernwasserversorgungen (ohne Abb.). Weitere Kennzahlen, die zur Beurteilung der Wasserverluste jedenfalls auch herangezogen werden sollten, sind die realen Verluste je Hausanschluss sowie der ILI (Infrastruktur-LeckverlustIndex). Die Beurteilung der Wasserverluste in Hinblick auf den Netzzustand und somit auf die Erneuerungsplanung sollte immer die Betrachtung aller genannten (bzw. verfügbaren) Wasserverlustkennzahlen beinhalten, da aufgrund unberücksichtigt gebliebener Einflussfaktoren ein fälschlich besseres oder schlechteres Abschneiden bei einer einzelnen Kennzahl nicht ausgeschlossen werden kann.

\subsection{Schadensraten und Leckkontrolle}

Zusätzlich sind für die Erneuerungsplanung die Schadenszahlen von Bedeutung. Auch hier gilt, dass zur Beurteilung, was als wenig oder viel anzusehen ist, nur Vergleiche gegenüber WVU mit ähnlichen Rahmenbedingungen gültig sein können. Wie die Wasserverluste unterliegen auch die Schadensraten den Einflussfaktoren Netzalter und Netzstruktur. Aus Abb. 3 ist ersichtlich, dass gerade hier die Einflussfaktoren unterschiedlich stark wirken. Während die Schadensraten bei den ländlich strukturierten Netzen wesentlich vom Alter beeinflusst sind, scheint das Alter bei den städtisch strukturierten Netzen eine untergeordnete Rolle $\mathrm{zu}$ spielen. Alleine der Umstand der höheren Urbanität (z. B. Bebauung, Verkehrslasten, sonstige Leitungsträger) verursacht auch bei jungen städtischen Leitungsnetzen eine höhere Schadensrate.

Bei der Bewertung der Schadenszahlen ist zudem zu beachten, dass die Zahl der gefundenen (und reparierten) Schäden nicht unbedingt der Zahl der tatsächlich vorhandenen Schäden entsprechen muss. Daraus erschließt sich der Zusammenhang mit der Intensität der Leckkontrolle. Wird intensiv und aktiv nach Lecks gesucht, kann davon ausgegangen werden, dass nur wenige größere Wasserverluste unentdeckt bleiben. Umgekehrt gilt, dass bei geringer Leckkontrolle durchaus Schäden unentdeckt bleiben können und somit die Schadensrate unterschätzt wird.

Eine Plausibilitätskontrolle der Schadenszahlen kann anhand der jährlich durchgeführten Leckkontrolle (z. B. ausgedrückt als Anteil der überprüften Leitungslängen) und der Wasserverluste erfolgen. Dabei können sich einige typische Zusammenhänge ergeben.

\subsection{Typische Zusammenhänge}

In Abb. 4 sind exemplarisch die Zusammenhänge von Wasserverlusten, Schadensraten und Intensität der Leckkontrolle bei der Netzzustandsbewertung beschrieben. Die Darstellung ist dabei weniger als quantitatives Werkzeug zu verstehen, sondern soll vielmehr den Dreieckszusammenhang zwischen diesen Kennzahlen verdeutlichen, die zur Beurteilung des Netzzustandes herangezogen werden.

Insbesondere die Bewertungen „mittlere bis hohe Wasserverluste“ in Kombination mit „geringe bis mittle- 


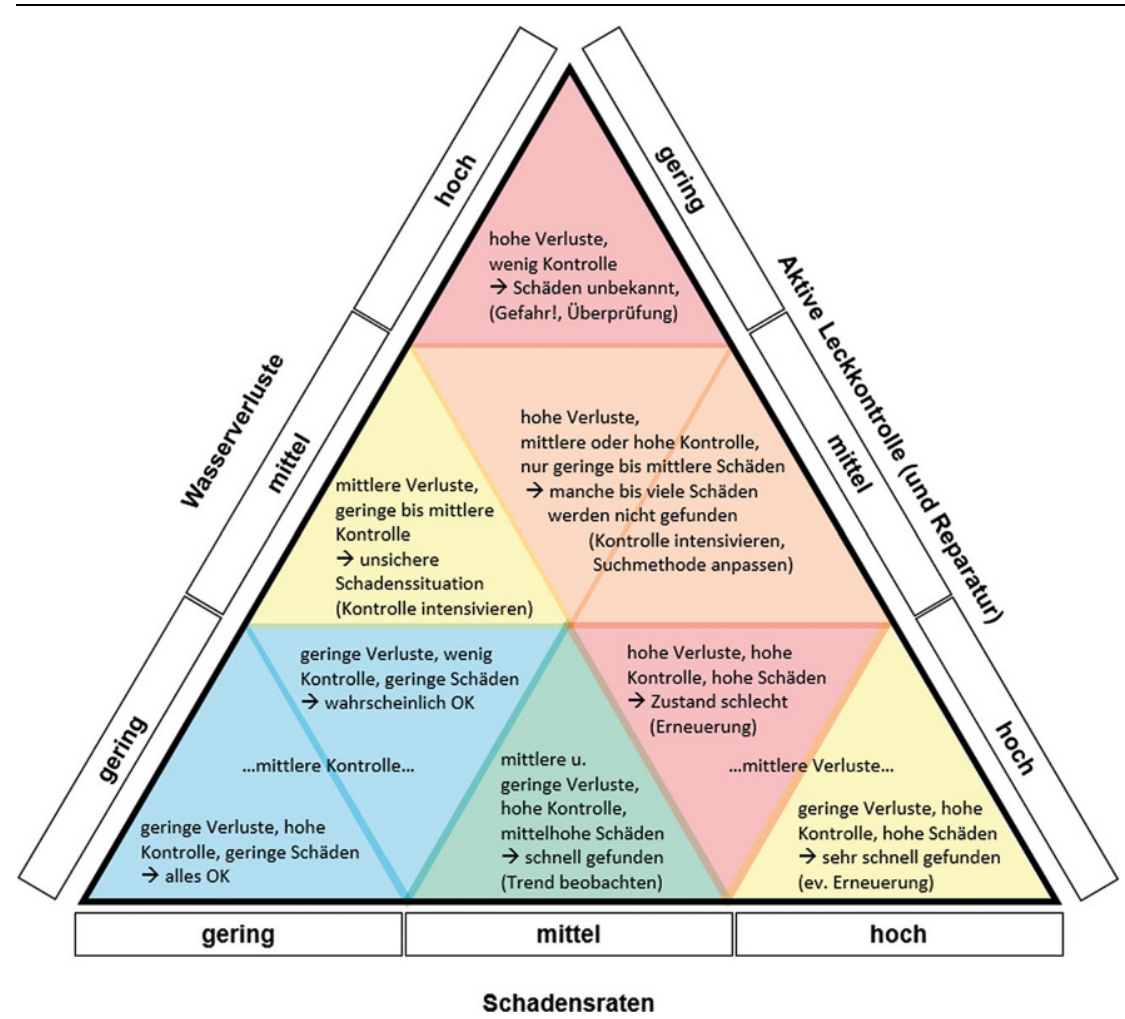

Abb. 4 Netzzustandsbewertung (Neunteufel et al. 2014)

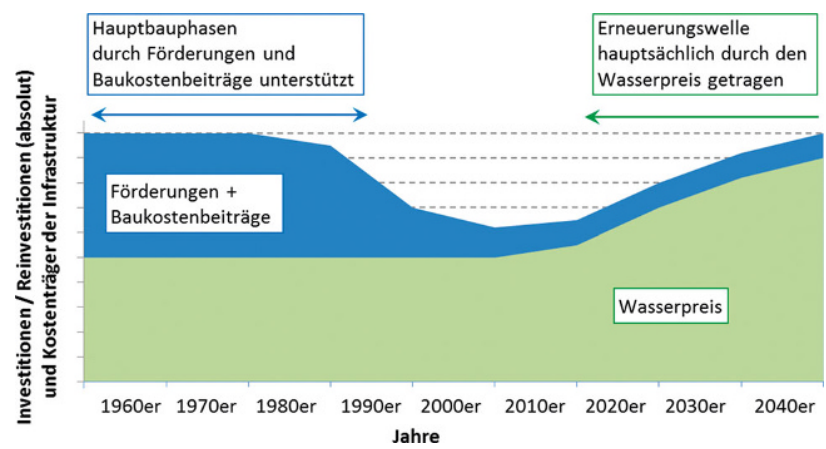

Abb. 5 Zusammensetzung der Finanzierung für Investitionen und Reinvestitionen in die Infrastruktur

re Intensität der Leckkontrolle" weist auf eine unsichere Schadenssituation hin, bei der die Zahl der gefundenen Schäden nicht unbedingt der Zahl der tatsächlich vorhandenen Schäden entspricht. Diesem Umstand sollte bei der Bewertung der Schadensraten in Hinblick auf die Erneuerungsplanung Rechnung getragen werden.

\subsection{Rehabilitationsraten}

Was bedeuten nun die beschriebenen Zusammenhänge für die Rehabilitationsraten?

Die Netzzustandsbeurteilung sollte immer nur innerhalb der jeweiligen gleichen Kennzahlenwerte bei anderen Leitungsnetzen auf eine kürzere Nutzungsdauer und eine frühere bzw. durchschnittlich höhere Rehabilitationserfordernis hinweisen.

Die Rehabilitationsraten der Wasserversorger, die am ÖVGW-Benchmarking teilgenommen haben und eine Netzalterquote von $50 \%$ oder älter aufweisen, betragen im Mittel knapp 0,7\% pro Jahr. Diese Rehabilitationsrate würde einer durchschnittlichen Nutzungsdauer von rund 140 Jahren entsprechen. Das ist natürlich eher unrealistisch. Die relativ geringe Rehabilitationsrate weist vielmehr darauf hin, dass viele WVU aufgrund der Errichtungszeitpunkte der Leitungsnetze bzw. des noch guten Netzzustandes erst am Anfang der ersten großen Erneuerungswelle stehen. Die Hauptbauphasen der österreichischen Wasserversorgungsnetze liegen entsprechend den Aufzeichnungen der Kommunalkredit Public Consulting (KPC) in den 1960er- bis 1990erJahren. In diesem Zeitraum wurden knapp $70.000 \mathrm{~km}$ der derzeit existierenden knapp $80.000 \mathrm{~km}$ der österreichischen Wasserleitungsinfrastruktur gebaut (KPC 2012).

Wenn aufgrund der Wasserverlustund Schadensentwicklung eine verstärkte Erneuerung noch nicht nötig erscheint, sollte die Zeit nach Möglichkeit genutzt werden, um Rücklagen für die Erneuerungswelle zu bilden.

\subsubsection{Rücklagen}

Die ursprünglichen Investitionen in die Infrastruktur der Wasserversorgung wurden noch durch Förderungen oder Baukostenbeiträge unterstützt. Die damals arbeitende (und steuerzahlende) Bevölkerung hatte somit ihre Infrastruktur in vollem Umfang bezahlt. Mittlerweile sind die Förderraten jedoch zurückgegangen und für zukünftige Reinvestitionen sind keine neuerlichen Baukostenbeiträge vorgesehen. Ein Wasserpreis, der in Anbetracht der existierenden Infrastruktur und der (noch) relativ geringen Erneuerungserfordernis derzeit gerade aufwandsdeckend ist, wird in naher Zukunft die Erneuerungswellen nicht finanzieren können. Empfindliche Preissteigerungen stehen daher spätestens dann an, wenn die Reinvestitionen in voller Höhe abschreibungswirksam werden und Zinsen für Kredite zurückgezahlt werden müssen. Abb. 5 zeigt dazu eine schematische, nicht maßstäbliche und 


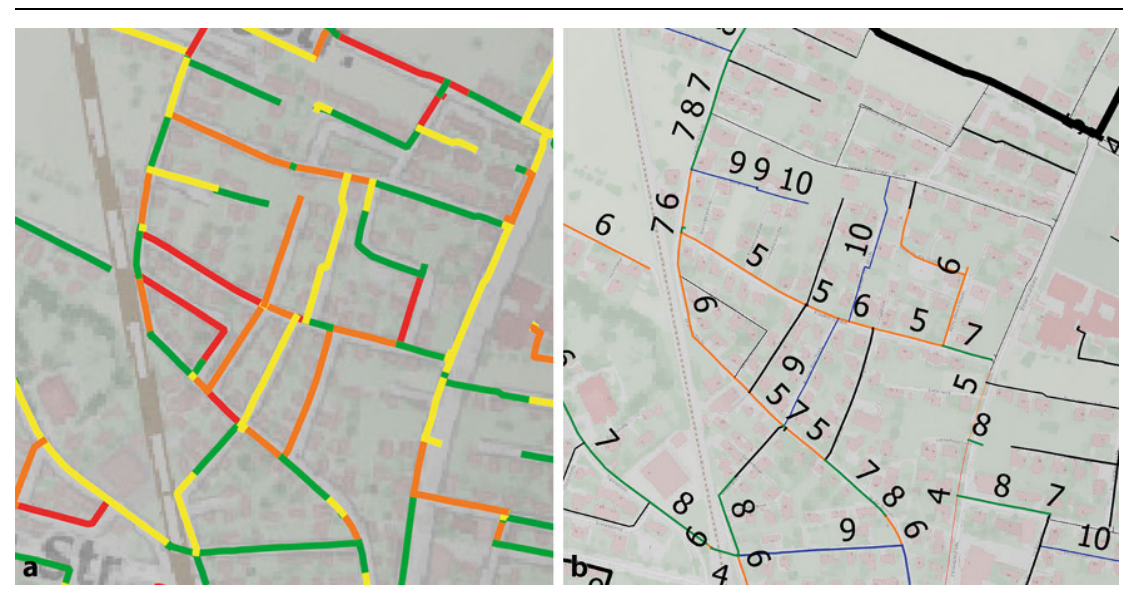

Abb. 6 Netzalter (links) und Erneuerungsprioritäten (rechts)

indexbereinigte Zusammensetzung der Finanzierung von Investitionen und Reinvestitionen in die Infrastruktur.

Rücklagenbildung kann helfen, die anstehenden Preissteigerungen etwas $\mathrm{zu}$ vergleichmäßigen, wenn aufgrund niedriger Schadenszahlen und niedriger Wasserverluste von einer Erneuerung im durchschnittlich erforderlichen Umfang noch abgesehen wird.

Da eine laufende Erneuerung theoretisch ab dem Zeitpunkt erfolgen müsste, zu dem Netzteile die erwartbare Lebensdauer erreicht haben und ausgetauscht werden müssten, wäre dies als Zeitpunkt für eine beginnende Rücklagenbildung denkbar.

Das Ausmaß der Rücklagen sollte in der Höhe dem Betrag entsprechen, mit dem die Erneuerung des Leitungsnetzes in erforderlichem Umfang zu marktüblichen Preisen abgegolten werden kann.

\subsubsection{Beispiel eines Rehabilitationsplans}

Wie ein konkreter Rehabilitationsplan aussehen kann und welche weiteren Einflussfaktoren existieren können, ist am besten anhand eines realen Beispiels nachzuvollziehen.

Zuerst wurde in einer GIS-basierten Karte die Altersstruktur des Leitungsnetzes dargestellt (Abb. 6 links). Dabei ist $\mathrm{zu}$ erkennen, dass in diesem Netz bereits ein bunter Mix verschiedener Altersstrukturen vorhanden ist. So sind ältere Netzbereiche (orange oder rot gekennzeichnet; entspricht NAX > $60 \%$ ) durch bereits erfolgte Erneuerungen immer wieder von jüngeren Abschnitten (grün und hellgrün; NAX $<40 \%$ ) unterbrochen.

In weiterer Folge wurden Prioritäten für die Erneuerung einzelner Stränge vergeben (Abb. 6 rechts). Je niedriger dabei die Zahl ist, desto früher sollte der entsprechende Leitungsteil ausgewechselt werden. Leitungen die noch keinen Zahlenwert zugewiesen bekom- men haben, stehen dabei noch nicht innerhalb des Planungszeitraumes zur Erneuerung an. Durch die automatisierte Auswertung der einzelnen Leitungsabschnitte, variieren auch die zugeordneten Zahlen z. B. innerhalb eines Straßenzugs, dies ist jedenfalls bei einer Detailplanung der einzelnen Bauabschnitte zu berücksichtigen.

Aufgrund des noch relativ guten Netzzustandes, beurteilt mittels Wasserverlusten und Schadensraten, wurden in dem konkreten Beispiel die höchsten Prioritäten anhand einer hydraulischen Rohrnetzmodellierung vergeben, die gezeigt hatte, welche Leitungen grundsätzlich verstärkt werden sollten. Erst in zweiter Linie wurde die Priorisierung nach Netzalter und Netzzustand vorgenommen.

Aus dem Vergleich von existierendem Netzalter und den gewählten Erneuerungsprioritäten wird jedenfalls ersichtlich, dass nicht immer die ältesten Netzteile als erstes erneuert werden müssen, sondern dass es weit mehr Einflussfaktoren zu berücksichtigen gilt.

Open access funding provided by University of Natural Resources and Life Sciences Vienna (BOKU).

Open Access Dieser Artikel wird unter der Creative Commons Namensnennung 4.0 International Lizenz (http:// creativecommons.org/licenses/by/4. $0 /$ deed.de) veröffentlicht, welche die Nutzung, Vervielfältigung, Bearbeitung, Verbreitung und Wiedergabe in jeglichem Medium und Format erlaubt, sofern Sie den/die ursprünglichen $\mathrm{Au}$ tor(en) und die Quelle ordnungsgemäß nennen, einen Link zur Creative Commons Lizenz beifügen und angeben, ob Änderungen vorgenommen wurden.
DVGW-Arbeitsblatt W392 (2003): Rohrnetzinspektion und Wasserverluste - Maßnahmen, Verfahren und Bewertungen. DVGW Regelwerk Technische Mitteilung, Mai 2003

KPC (2012): Investitionskostenerhebung Siedlungswasserwirtschaft 2012, https:/ /www. bmlfuw.gv.at/wasser/wasser-oesterreich/

foerderungen/trinkwasser_abwasser/aktuelle_ projekte/investitionskosten.html

(Abruf 6.2.2017)

Neunteufel, R., Mayr, E., Perfler, R. (2014): Rating-Triangle Water Loss, Failure Rate and Active Leakage Control; an evaluation scheme using Austrian and German guidelines and Austrian Benchmarking results/IWA 2014 Water Loss Conference, Vienna
Neunteufel R., Perfler, R., TheuretzbacherFritz, H. Kölbl, J. (2008): Explanatory factor 'Average Network Age index' (NAX) for mains failures and water losses. wami - Water Asset Management International 4.2 - JUNE 2008; ISSN (online): 1814-5442;@ IWA Publishing 2008 